\title{
Review of the anti-inflammatory effect of SIRT1 and SIRT2 modulators on neurodegenerative diseases
}

\author{
Yuqing Zhang a,b,c,1, Shailendra Anoopkumar-Dukie a,b,c,1, Devinder Arora a,b,c,1, Andrew K. Davey a,b,c,1,* \\ a Menzies Health Institute Queensland, Griffith University, Queensland, 4222, Australia \\ b School of Pharmacy and Pharmacology, Griffith University, Gold Coast Campus, Queensland, 4222, Australia \\ c Quality Use of Medicines Network, Queensland, 4222, Australia
}

\section{A R T I C L E I N F O}

\section{Keywords}

Sirtuin

SIRT1

SIRT2

Neuroinflammation

Neurodegeneration

Pharmacogenomics

\begin{abstract}
A B S T R A C T
Neurodegenerative disease refers to a range of chronic and progressive disorders that are characterized by dysfunction and loss of neurons. Neurodegeneration involves protein misfolding, oxidative injury, impaired mitochondrial function, neurotrophin deficiency and may also involve neuroinflammation. The sirtuin family of proteins plays a key role in this process suggesting that modulation of sirtuin can modify disease progression. This review examines experimental and clinical evidence relating to the potential role of SIRT1 and SIRT2, and their modulators in neurodegenerative diseases. Both neuroprotective effects and negative effects of SIRT1 activators, SIRT1 inhibitors and SIRT2 activators are discussed in a range of different disease models, including in vitro and in vivo Alzheimer's disease (AD), Parkinson's disease (PD), Huntingdon's disease (HD), multiple sclerosis (MS), amyotrophic lateral sclerosis (ALS). This highlights the potential of SIRT1 and SIRT 2 modulators as potential therapeutic agents. However, there is a paucity of clinical trials related to the effects of selective SIRT1 modulators, selective SIRT2 modulators or dual SIRT1/2 modulators on neuroinflammation and subsequent neurodegeneration.
\end{abstract}

\section{Introduction}

Neurodegenerative disease refers to a range of chronic and progressive disorders which are characterized by dysfunction and loss of neurons, and include Alzheimer's disease (AD), Parkinson's disease (PD) and multiple sclerosis (MS) (Amor et al., 2010). Neuronal damage or loss in focal areas of the central nervous system (CNS) directly leads to mobility functional impairment, cognitive impairment and/or depressive symptoms (Baquero and Martín, 2015; Ransohoff, 2016). According to the global burden of disease study, neurological disorders were the leading cause of disability and the second leading cause of death worldwide in 2016 (Feigin et al., 2019). In addition, global disability-adjusted life-years (DALYs) associated with AD, other dementias and PD steeply increased by more than 30\% from 2006 to 2016 (Hay et al., 2017). Therefore, there is a growing urgency for the investigation of novel treatments in neurodegenerative diseases.

\subsection{Pathophysiology of neurodegenerative diseases}

The basic pathogenetic mechanism of neurodegeneration is filamentous tangles and amyloid deposition (Skovronsky et al., 2006). The constituent of these misfolded protein aggregates varies from different diseases including amyloid $\beta$-protein (A $\beta$ ), tau ( $\tau$-protein), $\alpha$-synuclein in the central nervous system (Skovronsky et al., 2006). Extracellular $\mathrm{A} \beta$ deposits and intracellular tau aggregates in neurons are the main hallmarks of Alzheimer's disease (Spires-Jones et al., 2017). Tau in neurons and glia also contribute to tauopathy which is accountable for frontotemporal dementia (Spires-Jones et al., 2017). Alpha-synuclein accumulation which forms as Lewy bodies in neurons is the main pathologic feature of Parkinson's disease (Skovronsky et al., 2006). However, these different types of amyloid formation also present co-occurrence in each neurodegenerative disease (Spires-Jones et al., 2017). Other toxic proteins also play a key role in the neurodegeneration, for example, TAR DNA binding protein (TDP-43) in amyotrophic lateral sclerosis (ALS) and huntingtin protein expressed by the mu-

\footnotetext{
${ }^{*}$ Corresponding author. Menzies Health Institute Queensland, Griffith University, Queensland, 4222, Australia.

E-mail addresses: yuqing.zhang3@griffithuni.edu.au (Y. Zhang); s.dukie@griffith.edu.au (S. Anoopkumar-Dukie); d.arora@griffith.edu.au (D. Arora); a.davey@griffith.edu.au (A.K. Davey)

1 Postal address: School of Pharmacy and Pharmacology, Gold Coast Campus, Griffith University, QLD, 4222, Australia.
} 
tant gene (HTT) in Huntington's disease (Jimenez-Sanchez et al., 2017; Spires-Jones et al., 2017).

The propagation and accumulation of these misfolded proteins cause neurotoxicity and result in synaptic dysfunction and ultimately cell death. Oxidative injury, impaired mitochondrial function, and neurotrophin deficiency are also involved in neurodegeneration (Jellinger, 2010). Oxidative stress caused by protein aggregates can directly injure neurons and leads to DNA damage and structural alteration of proteins and lipids. It is considered that the imbalance between oxidative stress production and antioxidant defense is a vital hallmark of neurodegenerative diseases (Niedzielska et al., 2016). This feature of neurodegeneration has been simulated and identified in a number of experimental models. For instance, neurotoxins including 6-hydroxydopamine (6-OHDA), 1-methyl-4-phenyl-1,2,3,6-tetrahydropyridine (MPTP), and $\mathrm{A} \beta$ peptide can induce a high amount of oxidative species (Niedzielska et al., 2016).

Excessive and protracted neuroinflammation can directly influence neuronal apoptosis and thus result in acute neurological damage or neuron death and accelerate long-term neurodegeneration (Lyman et al., 2014). Sterile inflammation of the CNS is an outcome of neurological and psychological diseases which do not involve microorganisms as the trigger (Chen and Nuñez, 2010). Similar to microbially induced neuroinflammation, the characteristics of sterile inflammation include the recruitment of neutrophils and macrophages, and secretion of proinflammatory chemokines and cytokines such as TNF- $\alpha$ and IL-1 (Chen and Nuñez, 2010). The tumor necrosis factor family of cytokines such as TNF- $\alpha$ binds with TNF receptor and activates cell death pathways (Harry et al., 2008). As a result of sterile cell death, the inflammatory response, leukocyte infiltration and tissue injury are aggravated (Rock et al., 2010). Glial cells including astrocytes and microglia with varieties of inflammatory mediators also play a crucial role in neuroinflammation (Nicoll et al., 2010; Ransohoff, 2016). Neuroinflammation can also induce oxidative stress which is able to promote redox imbalance and stimulates the transcription of the pro-inflammatory mediators such as IL-1 $\beta$, IL-6, and TNF- $\alpha$ (Akbar et al., 2016).

\subsection{Sirtuins and neurodegeneration}

Sirtuin is a cluster of conserved proteins encoded by the SIRT genes in humans. They function as deacetylases to accomplish nicotinamide adenine dinucleotide (NAD + )-dependent deacetylation (Landry et al., 2000; Michan and Sinclair, 2007; Jiang et al., 2017). The sirtuin family consists of seven members including SIRT1, SIRT2, SIRT3, SIRT4, SIRT5, SIRT6, and SIRT7. The differences between sirtuin members in subcellular localization and tissue distribution are shown in Table 1. The sirtuin family regulates diverse physiologic activities such as maintenance of genomic stabilization, stress response, regulation of gluconeogenesis and fatty acid metabolism in individual organs (Finkel et al., 2009). The fact that sirtuins present the effect of delaying senescence has been generally acknowledged (Nakagawa and Guarente, 2014). The reason might be due to their dependence on NAD or influences on other systems such as glycolytic and glutamine metabolism (Nakagawa and Guarente, 2014). Following this, there has been interest in investigating the potential of sirtuin modulation in neurodegenerative diseases, which are often associated with the accumulation of senescent cells (Kritsilis et al., 2018).

The molecular mechanisms by which sirtuins may influence neurodegeneration are shown in Table 1 . Compelling evidence has indicated that sirtuins, especially SIRT1 and SIRT2, play an important role in neurodegenerative diseases. They have multiple targets such as NF- $\mathrm{kB}$, peroxisome proliferator-activator gamma coactivator $1-\alpha$ (PGC- $1 \alpha$ ), forkhead box protein O (FOXO) 1/3, and superoxide dismutase 2 (SOD2) (Kupis et al., 2016). Other sirtuins also target multiple path-
Table 1

Summary of the roles of SIRT1-7 in neurodegeneration.

\begin{tabular}{|c|c|c|c|}
\hline & $\begin{array}{l}\text { Subcellular } \\
\text { Localization }\end{array}$ & $\begin{array}{l}\text { Tissue } \\
\text { Distribution }\end{array}$ & $\begin{array}{l}\text { Disease Involvement and } \\
\text { Molecular Mechanisms }\end{array}$ \\
\hline SIRT1 & $\begin{array}{l}\text { Nucleus } \\
\text { Euchromatin } \\
\text { (Michan and } \\
\text { Sinclair, 2007) }\end{array}$ & $\begin{array}{l}\text { Liver, pancreas, } \\
\text { heart, muscle, } \\
\text { adipose tissue, } \\
\text { and brain } \\
\text { (especially } \\
\text { prefrontal cortex, } \\
\text { hippocampus, } \\
\text { basal ganglia, and } \\
\text { metabolic centers) } \\
\text { (Nogueiras et } \\
\text { al., 2012) }\end{array}$ & $\begin{array}{l}\text { SIRT1 presents protective } \\
\text { effects in neurodegeneration } \\
\text { through multiple mechanisms } \\
\text { including lowering amyloid } \beta \\
\text { (A } \beta \text { ) accumulation by reducing } \\
\text { its precursor protein and } \\
\text { enhancing } \alpha \text {-secretase activity. } \\
\text { SIRT1 deficiency is also } \\
\text { associated with the } \\
\text { aggregation of acetylated tau. } \\
\text { (Min et al., 2013) } \\
\text { SIRT1 has also been indicated } \\
\text { to deacetylate autophagy- } \\
\text { related components to reduce } \\
\alpha \text {-synuclein aggregates (Min } \\
\text { et al., 2013; Tang, 2017). } \\
\text { However, the overall } \\
\text { mechanism of SIRT1 in PD is } \\
\text { not clear. }\end{array}$ \\
\hline SIRT2 & $\begin{array}{l}\text { Cytoplasm } \\
\text { Shift to the } \\
\text { nucleus upon } \\
\text { stress and } \\
\text { mitosis (Michan } \\
\text { and Sinclair, } \\
2007 \text { ) }\end{array}$ & $\begin{array}{l}\text { Nervous tissue } \\
\text { (particularly in } \\
\text { the myelin } \\
\text { sheaths, pre- } \\
\text { myelinating cells, } \\
\text { and } \\
\text { oligodendrocytes), } \\
\text { adipose tissue, } \\
\text { muscle, heart, and } \\
\text { lung (Shoba et } \\
\text { al., 2009) }\end{array}$ & $\begin{array}{l}\text { SIRT2 inhibition was found to } \\
\text { attenuate the } \alpha \text {-synuclein } \\
\text { toxicity in in vivo or in vitro } \\
\text { models of PD via stimulating } \\
\text { the benign aggregation of } \alpha \text { - } \\
\text { synuclein toward acetylated } \\
\text { microtubule (Outeiro et al., } \\
2007 \text { ). SIRT2 is also } \\
\text { considered to influence } \\
\text { neuronal cholesterol } \\
\text { biosynthesis in in vivo HD } \\
\text { models (Donmez and } \\
\text { Outeiro, 2013). }\end{array}$ \\
\hline SIRT3 & $\begin{array}{l}\text { Mitochondria } \\
\text { (Michan and } \\
\text { Sinclair, 2007) }\end{array}$ & $\begin{array}{l}\text { Kidney, brain, } \\
\text { heart, liver, testes, } \\
\text { lung, ovary, } \\
\text { spleen, and } \\
\text { thymus } \\
\text { (Nogueiras et } \\
\text { al., 2012) }\end{array}$ & $\begin{array}{l}\text { SIRT3, } 4,5 \text { are associated with } \\
\text { mitochondrial function, energy } \\
\text { metabolism, and oxidative } \\
\text { stress (Gan and Mucke, } \\
\text { 2008). The mechanism of } \\
\text { them in neurodegeneration } \\
\text { needs further investigation. }\end{array}$ \\
\hline SIRT4 & $\begin{array}{l}\text { Mitochondria } \\
\text { (Michan and } \\
\text { Sinclair, 2007) }\end{array}$ & $\begin{array}{l}\text { Heart, brain, } \\
\text { kidney, liver, and } \\
\text { pancreas (Shoba } \\
\text { et al., 2009) }\end{array}$ & \\
\hline SIRT5 & $\begin{array}{l}\text { Mitochondria } \\
\text { (Michan and } \\
\text { Sinclair, 2007) }\end{array}$ & $\begin{array}{l}\text { Expressed in a } \\
\text { variety of tissue } \\
\text { (Du et al., } \\
2018 \text { ) }\end{array}$ & \\
\hline SIRT6 & $\begin{array}{l}\text { Nucleus } \\
\text { Heterochromatin } \\
\text { (Michan and } \\
\text { Sinclair, 2007) }\end{array}$ & $\begin{array}{l}\text { Widely expressed } \\
\text { in human tissues } \\
\text { (Michishita et } \\
\text { al., 2005) }\end{array}$ & $\begin{array}{l}\text { SIRT6 may contribute to DNA } \\
\text { repair in aging (Jesko et al., } \\
\text { 2017). }\end{array}$ \\
\hline SIRT7 & $\begin{array}{l}\text { Nucleolus } \\
\text { (Michan and } \\
\text { Sinclair, 2007) }\end{array}$ & $\begin{array}{l}\text { Widely expressed } \\
\text { in human tissues } \\
\text { (Michishita et } \\
\text { al., 2005) }\end{array}$ & Limited information \\
\hline
\end{tabular}

ways that might eventually affect cellular homeostasis and subsequent neurodegeneration (Kupis et al., 2016). However, due to limited data in relation to SIRT3-7, this review will focus on the effects of SIRT1 and SIRT2 modulators on neuroinflammation and neurodegenerative conditions.

\subsection{Polymorphisms in the SIRT gene in the population}

Genetic polymorphism is defined as the occurrence of at least two alleles at a locus with a population frequency greater than 1\% (Daly, 2010). When the DNA sequence differs at a single base, it is defined as a single nucleotide polymorphism (SNP) (Shaw, 2013). Some polymorphisms can lead to functionally significant effects in drug metabo- 
Table 2

Summary of the effects and mechanisms of resveratrol (SIRT1 activator) on neurodegenerative diseases.

\begin{tabular}{|c|c|c|}
\hline Condition & $\begin{array}{l}\text { Possible SIRT1 } \\
\text { activator-Induced } \\
\text { Mechanisms }\end{array}$ & Effect \\
\hline Neuroinflammation & $\begin{array}{l}\downarrow \text { MMP-9, iNOS, IL-1 } \beta, \\
\text { IL-6 (BV-2 + PC12 cell } \\
\text { line) (Ye et al., 2013); } \\
\downarrow \text { acetylated p53, } \\
\text { cleaved caspase-3 (N9 } \\
\text { cell line) (Li et al., } \\
2015 \text { ). }\end{array}$ & $\begin{array}{l}\text { Resveratrol has shown to } \\
\text { suppress neuroinflammation } \\
\text { and attenuate apoptosis. }\end{array}$ \\
\hline Alzheimer's disease & $\begin{array}{l}\downarrow \text { iNOS, COX-2 (primary } \\
\text { astrocytes) (Scuderi et } \\
\text { al., 2014); } \\
\downarrow \text { ROCK1 activity (PC12) } \\
\text { (Feng et al., 2013); } \\
\downarrow \text { NF-kB/SIRT1 (BV2) } \\
\text { (Chen et al., 2005); } \\
\uparrow \text { SIRT1, AMPK (SAMP8 } \\
\text { mouse) (Porquet et } \\
\text { al., 2013); } \\
\downarrow \text { acetylated } \\
\text { PGC-1 } \alpha / \text { P53 (p25 } \\
\text { transgenic mouse) (Kim } \\
\text { et al., 2007); } \\
\downarrow \text { MMP-9, amyloid- } \beta \\
\text { accumulation } \\
\text { (retrospective clinical } \\
\text { study) (Moussa et al., } \\
\text { 2017). }\end{array}$ & $\begin{array}{l}\text { Resveratrol can extend the } \\
\text { lifespan of } \mathrm{AD} \text { mouse models } \\
\text { and decreased mutant protein } \\
\text { aggregation. }\end{array}$ \\
\hline Parkinson's disease & $\begin{array}{l}\text { Altered autophagy } \\
\text { activity (MPTP-induced } \\
\text { mouse) (Guo et al., } \\
2016 \text { ); } \\
\uparrow \text { SIRT1/AMPK/PGC-1 } \alpha \\
\text { (skin fibroblast carrying } \\
\text { PARK2, dopaminergic } \\
\text { SN4741 cells) (Ferretta } \\
\text { et al., 2014); } \\
\downarrow \text { RAS, AII/ATI/NADPH- } \\
\text { oxidase activity (aged } \\
\text { rats, dopaminergic } \\
\text { MES23.5 cells and N9 } \\
\text { cells) (Diaz-Ruiz et } \\
\text { al., 2015). }\end{array}$ & $\begin{array}{l}\text { Resveratrol can decrease } \\
\text { dopaminergic neuron loss and } \\
\text { ameliorate motor impairment. }\end{array}$ \\
\hline $\begin{array}{l}\text { Amyotrophic } \\
\text { lateral sclerosis, } \\
\text { multiple sclerosis, } \\
\text { aging }\end{array}$ & $\begin{array}{l}\uparrow \text { Mitochondrial } \\
\text { function (SOD1 } \\
\text { transgenic VSC4.1 } \\
\text { motor neuron-like cells) } \\
\text { (Wang et al., 2011); } \\
\uparrow \text { SIRT1/AMPK } \downarrow \text {, } \\
\text { PGC-1 } \propto \text {, P53 acetylation } \\
\text { (SOD1 transgenic } \\
\text { mouse, aged mouse) } \\
\text { (Mancuso et al., } \\
2014 \text {; Song et al., } \\
2014 \text {; Moorthi et al., } \\
2015 \text { ); } \\
\downarrow \text { ROS (retinal ganglion } \\
\text { cells) (Khan et al., } \\
\text { 2012); } \\
\downarrow \text { neuroinflammation } \\
\text { (isoflurane-induced aged } \\
\text { mouse) (Li et al., } \\
\text { 2014). }\end{array}$ & $\begin{array}{l}\text { Resveratrol can extend the } \\
\text { lifespan of ALS mouse models, } \\
\text { decrease cellular apoptosis, } \\
\text { improve cognitive impairment, } \\
\text { and inhibit neuroinflammation } \\
\text { in aged rats, show protection in } \\
\text { MS models. }\end{array}$ \\
\hline
\end{tabular}

lism, which explains the inter-individual variation in drug response to some extent (Daly, 2010). This concept of pharmacogenetics has been used in genetic-guided drug discovery and development. Researchers have found SIRT1 gene polymorphisms in cholesterol metabolism, cardiac diseases, breast, and lung cancer (Shimoyama et al., 2012; Rizk et al., 2016; Lv et al., 2017). SIRT gene polymorphisms in popula-
Table 3

Summary of the effects and mechanisms of SIRT2 inhibitors on neurodegenerative diseases.

\begin{tabular}{|c|c|c|}
\hline Condition & $\begin{array}{l}\text { Possible SIRT2 Inhibitor- } \\
\text { Induced Mechanisms }\end{array}$ & Effect \\
\hline Neuroinflammation & $\begin{array}{l}\downarrow \text { Neuroinflammation, TNF- } \alpha \text {, } \\
\text { IL- } 6 \text { expression, altered } \\
\text { translocation of NF-кB (BV2) } \\
\text { (Wang et al., 2016); } \\
\uparrow \text { acetylation of } \alpha \text {-tubulin, } \\
\text { autophagy (primary rat } \\
\text { astrocytes) (Scuderi et al., } \\
2014 \text { ); }\end{array}$ & $\begin{array}{l}\text { AGK2 has shown to } \\
\text { protect neurons from the } \\
\text { toxicity of mutant toxin } \\
\text { aggregation. }\end{array}$ \\
\hline Parkinson's disease & $\begin{array}{l}\uparrow \text { Acetylation of } \alpha \text {-tubulin, } \\
\text { maintain redox network } \\
\text { (MPTP-induced mouse, } \\
\text { rotenone-induced rat) (Wang } \\
\text { et al., 2015; Guan et al., } \\
2016 \text { ); } \\
\downarrow \alpha \text {-synuclein-induced } \\
\text { cytotoxicity, } \uparrow \text { microtubule } \\
\text { stabilization (primary } \\
\text { midbrain culture, LUHMES } \\
\text { cells, H4 neuroglioma cells) } \\
\text { (Outeiro et al., 2007; Chen } \\
\text { et al., 2015a). }\end{array}$ & $\begin{array}{l}\text { AK-7 could attenuate } \\
\text { behavioral abnormality in } \\
\text { aged mice. AGK2 and } \\
\text { AK-1 also could decrease } \\
\alpha \text {-synuclein toxicity and } \\
\text { protect dopaminergic } \\
\text { neurons. }\end{array}$ \\
\hline Alzheimer's disease & $\begin{array}{l}\uparrow \text { Acetylation of } \alpha \text {-tubulin, } \uparrow \\
\text { autophagy function (cybrids of } \\
\text { SH-SY5Y and platelets from } \\
\text { sporadic AD patients) (Silva } \\
\text { et al., 2016). }\end{array}$ & $\begin{array}{l}\mathrm{A} \beta \text { aggregates level could } \\
\text { be decreased by AK1 } \\
\text { treatment in vitro. }\end{array}$ \\
\hline $\begin{array}{l}\text { Huntingtin's } \\
\text { disease }\end{array}$ & $\begin{array}{l}\downarrow \text { Mutant Huntingtin toxicity } \\
\text { (primary striatal neuron), } \downarrow \\
\text { SREBP-2/ } \downarrow \text { sterol synthesis/ } \downarrow \\
\text { neurodegeneration (fly HD } \\
\text { models) (Luthi-Carter et al., } \\
2010 \text { ); } \\
\downarrow \text { neuronal atrophy (HD } \\
\text { mouse) (Chopra et al., } \\
2012 \text { ). }\end{array}$ & $\begin{array}{l}\text { AK1, AGK2, and AK-1 } \\
\text { showed neuroprotection } \\
\text { in fly and mouse HD } \\
\text { models. }\end{array}$ \\
\hline
\end{tabular}

tions suffering from neurological or psychological diseases still remain unclear and need further investigation.

In a case-control pilot study of age-related macular degeneration (AMD) with 253 subjects in the patient group and 292 subjects in the control group, the distribution of an SNP of SIRT1, rs12778366 allele, showed significant differences between AMD group and control group (Chen et al., 2015b). This study also indicated that homozygotes of a minor allele (base C) of SIRT1 had higher odds of AMD morbidity. Due to the scope and depth of this study, the relationship between variants in rs12778366 and the regulation of SIRT1 expression could not be elucidated. In contrast, minor allele carriers of rs12778366 have better glucose tolerance and a longer life span in the Dutch population (Figarska et al., 2013). Apparently, the variation of the genotype of this allele including $\mathrm{T} / \mathrm{T}, \mathrm{T} / \mathrm{C}$, and $\mathrm{C} / \mathrm{C}$ can affect down-stream activities and subsequent SIRT1 expression. It is clear that the impact of rs12778366 in different disease contexts warrants further investigation.

Two cohorts of case-control studies found that the SIRT2 rs10410544 $\mathrm{T}$ allele may have the potential to increase the risk of Alzheimer's disease in Caucasian populations (Polito et al., 2013). Another study of the Han Chinese population also showed that rs10410544 C/T genotype was associated with late-onset Alzheimer's disease (Xia et al., 2014). The function of this polymorphism still remained unknown according to these studies.

Based on the current evidence, changes in SIRT1, 2 functionality due to polymorphisms might be associated with neurodegenerative conditions. SIRT1, 2 enhancement or suppression in people with these polymorphisms may potentially have benefits in delaying these conditions. 


\section{Findings of SIRT1 and SIRT 2 modulators on neurodegenerative conditions}

\subsection{Resveratrol}

Resveratrol has long been considered as a natural SIRT1 activator and has several purported effects on neuroinflammatory diseases (Table 2). There are views that its activation effect on SIRT1 might be exerted via stimulating 5' AMP-activated protein kinase (AMPK) and peroxisome proliferator-activated receptor $\delta$ (PPAR $\delta$ ) pathways (Cheng et al., 2019). However, there is also literature indicating that resveratrol has a direct structure-activity relationship with SIRT1. Fig. 1 shows the chemical structure of resveratrol. Allosteric mechanism studies have elucidated that resveratrol is able to strengthen the "loose-binding" of SIRT1 and substrates containing bulky hydrophobic groups (Hou et al., 2016). This SIRT1 activation needs three resveratrol molecules to change the conformation of the N-terminal domain (NTD) of SIRT1 (Ovesna and Horvathova-Kozics, 2005; Cao et al., 2015; Hou et al., 2016). In addition to the potential of resveratrol to improve the binding of SIRT1 to its substrates, there is also evidence that resveratrol enhances the expression of SIRT1 (Ye et al., 2013). This is further discussed in the following sections.

\subsubsection{Neuroinflammation}

Resveratrol has been found to suppress the inflammation in lipopolysaccharide (LPS)-induced microglial BV-2 cells and protect neuronal PC12 cells from caspase-3-dependent apoptosis by increasing the expression of SIRT1 and deacetylation level of tumor suppressor p53 (Ye et al., 2013). SIRT1 knockdown in microglial N9 cells attenuated resveratrol's down-regulation effect on MMP-9, IL-1 $\beta$, and IL-6 (Li et al., 2015). Matrix metalloproteinases (MMPs) contribute to disrupting the integrity of the blood-brain-barrier (BBB) (Rosenberg, 2002), which allows IL-1 $\beta$, IL-6, and other cytokines originating from the peripheral immune system permeate to influx into the brain parenchyma (Wong et al., 2004). Resveratrol has been found to inhibit MMPs (Pandey et al., 2015) as well as reduce production of a range of cytokines (Frozza et al., 2013; de Sá Coutinho et al., 2018), which indicates the potential for a multi-faceted influence on neuroprotection. In a retrospective clinical study, resveratrol was used as a SIRT1 activator to treat mild-moderate $\mathrm{AD}$ patients for 52 weeks (Moussa et al., 2017). The result showed that resveratrol could decrease the level of MMP-9 and stabilize the level of A $\beta 40$ in cerebrospinal fluid (CSF) and attenuate disease aggravation (Moussa et al., 2017). However, whether the underlying mechanism is in relation to SIRT1 or its other pleiotropic effect has not been confirmed.

\subsubsection{Alzheimer's disease}

Resveratrol has been indicated to improve cellular viability, inhibit inflammation and suppress the toxicity of aggregation of misfolded amyloid- $\beta$ in in vitro studies. As mentioned above, excessive accumulation of amyloid $\beta$-protein is able to cause oxidative stress, cytotoxicity, subsequent synaptic dysfunction and neuron loss in the brain of $\mathrm{AD}$ patients (Huang et al., 2016). In Scuderi's study, resveratrol suppressed the proliferation and activation of primary astrocytes and inhibited the expression of inducible nitric oxide synthase (iNOS) and cyclooxygenase-2 (COX-2) induced by A $\beta$ 1-42 treatment (Scuderi et al., 2014). Resveratrol also was found to protect PC12 cells from A $\beta$ 25-35 neurotoxicity and apoptosis through upregulating SIRT1 expression. Based on the result, this study also hypothesized that resveratrol as a SIRT1 activator could further downregulate the expression of Rho-associated kinase 1 (ROCK1) (Feng et al., 2013). ROCK1 has been reported to participate in enhancing amyloid- $\beta$ protein accumulation (Henderson et al., 2016). Both overexpression of SIRT1 and resveratrol can suppress NF- $\mathrm{KB}$ pathway induced by A $\beta$ 1-42 in cultured microglial BV2 cells and attenuate the $A \beta$ toxicity in primary cortical cultures (Chen et al., 2005). $\mathrm{NF}-\mathrm{\kappa B}$ plays a crucial role in regulating the immune response to inflammation in the nervous system. Numerous chemokines, cytokines, enzymes and other molecules or factors in inflammation process are regulated by NF- $\mathrm{KB}$ (Shih et al., 2015). This evidence indirectly indicated that the protective effect of resveratrol in $\mathrm{AD}$ models has a linkage with SIRT1 activity.

In in vivo $\mathrm{AD}$ studies, resveratrol also protected $\mathrm{AD}$ models, neurotoxic p25 transgenic mice, from CNS degeneration and cognitive decline via up-regulation of SIRT1 (Kim et al., 2007). The acetylation level of SIRT1 substrates peroxisome proliferator-activated receptor-gamma coactivator (PGC)- $1 \alpha$ and tumor suppressor p53 were also down-regulated, which indirectly verified resveratrol's SIRT1 modulation (Kim et al., 2007). PGC- $1 \alpha$ and p53 both play important roles in mitochondria biogenesis, oxidative response, and apoptosis (Chang et al., 2012; Sweeney and Song, 2016). The lifespan and cognitive impairment of age-accelerated mouse models (SAMP8) improved and amyloid deposition was also attenuated through dietary resveratrol (Porquet et al., 2013). SIRT1 and AMPK elevation by resveratrol have also been observed in mouse brain samples (Porquet et al., 2013).

\subsubsection{Parkinson's disease}

Resveratrol has been found to alleviate dopaminergic neuron loss, the decrease of tyrosine hydroxylase and dopamine levels in neurotoxin/mitochondrial toxin 1-methyl-4-phenyl-1, 2, 3, 6-tetrahydropyridine (MPTP)-induced PD mouse models (Guo et al., 2016).<smiles>Oc1ccc(/C=C/c2cc(O)cc(O)c2)cc1</smiles>

roveratrol<smiles>N#C/C(=C\c1ccc(-c2cc(Cl)ccc2Cl)o1)C(=O)Nc1cccc2ncccc12</smiles>

ACK2
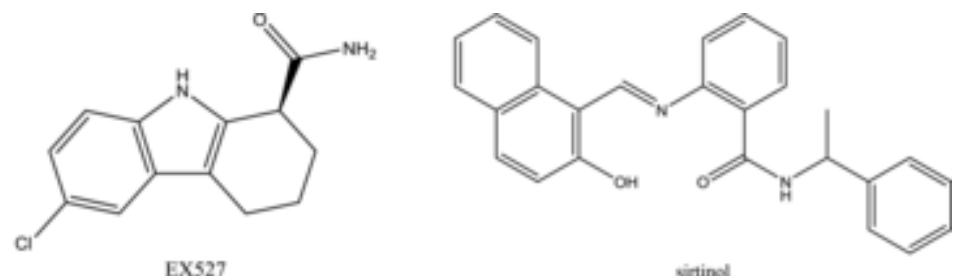

EX527

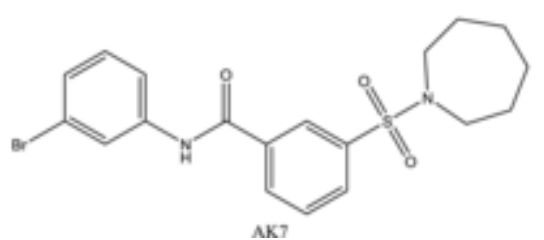

AK7 
This study suggested that SIRT1 participated in ameliorating motor impairment and pathological changes via autophagy degradation of $\alpha$-synuclein (Guo et al., 2016). Autophagy participates in the degradation of damaged organelles such as mitochondria, endoplasmic reticulum, and peroxisomes, as well as eliminating intracellular pathogens and recycling of cellular metabolites (Glick et al., 2010). This process can be initiated in response to a pathogenic insult to enclose the infection and promote the clearance of the pathogen (Shi et al., 2012).

Resveratrol has also been shown to promote mitochondrial oxidative capacity in a skin fibroblast PD model carrying pathogenic mutation PARK2 (Ferretta et al., 2014). The activation of SIRT1, AMPK, and PGC- $1 \alpha$ pathway was demonstrated to be the possible mechanism by determining the activity of their downstream targets (Ferretta et al., 2014). Mudo's study similarly reported that resveratrol protected dopaminergic SN4741 cells from death after MPTP treatment with an increase of PGC-1 $\alpha$ and SIRT1 levels (Mudo et al., 2012). AMPK and SIRT1 have a role as metabolic sensors and affect PGC- $1 \alpha$ as part of a network that helps to maintain metabolic homeostasis (Canto and Auwerx, 2009). This is pertinent given that the alteration of cell metabolism, including redox homeostasis and bioenergetics, strongly contributes to the neurodegeneration of dopaminergic cells in PD (Anandhan et al., 2017).

The brain renin-angiotensin system (RAS) also influences learning and memory function, cognition and the aging process (Wright and Harding, 2004; Diz et al., 2008; Mogi et al., 2012). Angiotensin stimulation can induce oxidative stress, neuroinflammation and cellular aging in the brain, and result in cognitive impairment and subsequent neurodegeneration (Mogi et al., 2012). RAS was found to be over-activated in the nigrostriatal system of aged rats, potentially leading to PD (Diaz-Ruiz et al., 2015). The SIRT1 activation effect of resveratrol has been found to inhibit angiotensin II (AII)/AII type 1 receptors (AT1)/NADPH-oxidase activity in aged rats and cultured dopaminergic MES 23.5 cells and microglial N9 cells (Diaz-Ruiz et al., 2015).

\subsubsection{Amyotrophic lateral sclerosis, multiple sclerosis, and aging}

In mutant superoxide dismutase 1 (SOD1)-transgenic ALS in vitro models, resveratrol has been shown to increase the viability of VSC4.1 motor neuron-like cells and improve mitochondria function via up-regulating SIRT1 (Wang et al., 2011). In SOD $1^{\mathrm{G} 93 \mathrm{~A}}$ transgenic mouse models, researchers also found resveratrol inhibited the apoptosis of motor neurons and extended the lifespan through multiple pathways including SIRT1 and AMPK activation, stabilizing autophagy, suppressing oxidative stress and P53 acetylation (Mancuso et al., 2014; Song et al., 2014).

Resveratrol was also able to decrease apoptosis and upregulate SIRT1 expression in the brain samples of aged rats (24 months old) but failed to improve learning and memory function (Moorthi et al., 2015). However, resveratrol could alleviate isoflurane-induced cognitive impairment of aged mice and inhibit neuroinflammation and apoptosis in the hippocampus in another study (Li et al., 2014). The combined treatment of two SIRT1 activators (resveratrol and SRTAW04) has been shown to improve cell viability and alleviated ROS and repress damage of mitochondrial membrane in retinal ganglion cells model which simulates the optic neuritis which is a symptom of multiple sclerosis (Khan et al., 2012).

Conversely, Markert's research could not find positive evidence in relation to resveratrol in ALS mouse models (Markert et al., 2010).

\subsection{EX527, sirtinol}

EX527 exerts an inhibitory effect on SIRT1 by depriving its $\mathrm{NAD}^{+}$-dependent deacetylation catalysis (Gertz et al., 2013). The structure of EX527 is shown in Fig. 1. EX527 has been shown to aggravate tau accumulation through increasing acetylation and decreasing nation of tau in primary neurons and transgenic HEK293T cells respectively (Min et al., 2010). It has been demonstrated that the elevation of tau acetylation can repress tau polyubiquitination, which mediates protein degradation (Min et al., 2010). However, in Smith's research, EX527 improved voluntary movement and brain pathological impairment in transgenic R6/2 mouse models of Huntington's disease (Smith et al., 2014). In addition, an exploratory double-blind, randomized clinical trial of EX527 on early-stage HD patients conducted by Sussmuth and colleagues suggested that EX527 is safe and tolerated in early-stage HD patients (Sussmuth et al., 2015). However, there was no analysis of biological samples. EX527 was reported to protect the transgenic neuronal cells from mutant SOD1 toxicity and increase the viability of neurons in the transgenic SH-SY5Y cell model of amyotrophic lateral sclerosis (Valle et al., 2014).

Sirtinol was originally reported to confer the SIRT2 inhibition effect in 2001 (Grozinger et al., 2001). Following that, its analogs exerting SIRT1 and SIRT2 inhibition were synthesized and evaluated (Mai et al., 2005). Sirtinol has been reported to reverse the protective effect of resveratrol in 6-hydroxydopamine (6-OHDA) or $\alpha$-synuclein induced neurodegeneration in differentiated SK-N-BE cell models (Albani et al., 2009). This indirectly indicated sirtinol's SIRT1 inhibitory effect. However, series of SIRT1 inhibitors have already been designed and synthesized based on varieties of methodologies (Hu et al., 2014; Jiang et al., 2017). More pharmacological studies need to be conducted to evaluate and compare their biological effects in neurodegenerative conditions.

\subsection{SIRT2 inhibitors}

The pharmacological evaluation of existing SIRT2 inhibitors such as AGK2, AK-7, and AK1 has been explored in different neurodegenerative models (Table 3). Their structures are shown in Fig. 1. AGK2 inhibited the activation of BV2 microglia induced by LPS and reduced the mRNA levels of TNF- $\alpha$ and IL-6. It also prevented the nuclear translocation of NF-kB (Wang et al., 2016). AGK2 was also indicated to reduce the activation of primary rat astrocytes and subsequent release of pro-inflammatory factors (Scuderi et al., 2014). However, it has also been reported that SIRT2 inhibition could compound neuroinflammation stimulated by traumatic brain injury in mouse models (Yuan et al., 2016).

SIRT2 inhibitors showed a neuroprotective effect on PD models. AK-7 can attenuate behavioural abnormality in aging mice after MPTP treatment via increasing acetylation of $\alpha$-tubulin and regulating redox balance (Guan et al., 2016). A similar result was also found in another rotenone-induced PD rat model (Wang et al., 2015). Dopamine depletion and neuron loss were also found to be decreased by AK-7 treatment in MPTP-induced PD mouse models (Chen et al., 2015a). In primary midbrain cultures, AGK2 and AK-1 protected dopaminergic cells from death induced by $\alpha$-synuclein toxicity (Outeiro et al., 2007). AK-7 and AGK2 also ameliorated $\alpha$-synuclein cytotoxicity in transgenic human fetal LUHMES cells and transgenic $\mathrm{H} 4$ neuroglioma cells respectively (Outeiro et al., 2007; Chen et al., 2015a). The mechanism involved might be elevated acetylation of $\alpha$-tubulin by SIRT2 inhibition increasing the affinity of $\alpha$-synuclein binding to microtubules, which results in reduced neurotoxicity (Outeiro et al., 2007).

Silva's team discovered that AK1 improved the acetylation level of tubulin, autophagy vesicular completion and the A $\beta$ aggregation in $\mathrm{AD}$ cell models (differentiated cybrids of SH-SY5Y cells and platelets from sporadic AD subjects) (Silva et al., 2016). Increased acetylated tubulin/ $\alpha$-tubulin ratio was also found in the postmortem samples of $\mathrm{AD}$ brains. They proposed that acetylation of tubulin induced by SIRT2 inhibition could enhance autophagic trafficking and exert subsequent protective effects (Silva et al., 2016). 
In Huntington's disease models, AK1 and AGK2 have been shown to protect the primary rat striatal neuron from mutant Huntingtin toxicity and decrease neurodegeneration in Drosophila HD models through down-regulating sterol biosynthesis (Luthi-Carter et al., 2010). This study also proposed a possible mechanism whereby $\alpha$-tubulin acetylation by SIRT2 inhibition suppresses sterol regulatory element-binding protein (SREBP-2) translocation (Luthi-Carter et al., 2010). AK-7 also promotes motor function and reverses neuronal atrophy of HD mouse models (Chopra et al., 2012).

\section{Discussion}

In this review, we have discussed the growing evidence that SIRT1 and SIRT2 play important roles in the pathophysiology of neurodegenerative diseases. This includes the discovery of SIRT1 and SIRT2 gene polymorphisms and their impact on neurodegenerative diseases which indicates the potential value of the application of SIRT1 and SIRT2 modulators. Further to this, we reviewed the pharmacological studies of SIRT1 and SIRT2 modulators in different neurodegeneration models.

Understanding drug-protein catalytic relationships and 3D structure-based design are fundamental for exploring and designing SIRT1 and SIRT2 drugs (Jiang et al., 2017). In spite of that, resveratrol is often considered to work through pleiotropic effects (Cheng et al., 2019). However, the fact that there is evidence linking resveratrol to SIRT1 activity and the allosteric mechanisms involved via SIRT1 activation should not be overlooked (Cao et al., 2015). SIRT1 activation by resveratrol has been shown to improve neurodegeneration through attenuating neuroinflammation and oxidative stress induced by amyloid deposition (Porquet et al., 2013). Potential pathways include PGC- $1 \alpha$ and $\mathrm{P} 53$ in mitochondria biogenesis and oxidative response, and NF- $\mathrm{KB}$ in inflammation (Chen et al., 2005; Kim et al., 2007). The interaction between AMPK and SIRT1 is also involved (Ferretta et al., 2014; Mancuso et al., 2014). Regarding SIRT1 inhibition, EX527 showed protective effects in HD and ALS models (Smith et al., 2014; Valle et al., 2014). However, negative results of EX527 and sirtinol indicate that more investigation is needed.

In contrast to SIRT1 activation, the inhibition of SIRT2 appears to be another potential approach to treat neurodegeneration. SIRT2 inhibitors can decrease neuroinflammation and cytotoxicity induced by toxins or mutant protein aggregation (Outeiro et al., 2007; Scuderi et al., 2014; Guan et al., 2016; Wang et al., 2016). Furthermore, acetylation of $\alpha$-tubulin also seems to be an important mechanism to exert neuroprotection. Elevated acetyl- $\alpha$-tubulin can improve the microtubule network, which influences protein aggregation, microtubule-dependent trafficking of autophagic vesicles and SREBP-2 (Luthi-Carter et al., 2010; Silva et al., 2016). This subsequently affects down-stream autophagy activities and sterol biosynthesis respectively to generate neuroprotection (Luthi-Carter et al., 2010; Silva et al., 2016).

Based on the pharmacologic studies, SIRT1 and SIRT2 modulators show a promising approach to target the main pathological problems combining inflammation, oxidative stress, and misfolded protein aggregation. More clinical trials related to SIRT1 and SIRT2 modulators need to be conducted to evaluate the safety status and therapeutic effects on human subjects of neurodegenerative diseases. Systematic research investigating and comparing the effects, structure-activities relationships, selectivity, and relative potencies of SIRT1 modulators, SIRT2 modulators or dual SIRT1/2 modulators on neurodegeneration is also needed to facilitate the design and synthesis of more potent and selective SIRT1 and SIRT2 modulators.

\section{Author contributions}

Andrew K. Davey, Shailendra Anoopkumar-Dukie, Devinder Arora and Yuqing Zhang designed this study. All authors contributed to its re- vision and preparation of the final manuscript.

\section{Declaration of competing interest}

All authors declare no conflicts of interest.

\section{References}

Akbar, M., Essa, M.M., Daradkeh, G., et al., 2016. Mitochondrial dysfunction and cell death in neurodegenerative diseases through nitroxidative stress. Brain Res. 1637, 34-55.

Albani, D., Polito, L., Batelli, S., et al., 2009. The SIRT1 activator resveratrol protects SK-N-BE cells from oxidative stress and against toxicity caused by alpha-synuclein or amyloid-beta (1-42) peptide. J. Neurochem. 110, 1445-1456.

Amor, S., Puentes, F., Baker, D., et al., 2010. Inflammation in neurodegenerative diseases. Immunology 129, 154-169.

Anandhan, A., Jacome, M.S., Lei, S., et al., 2017. Metabolic dysfunction in Parkinson's disease: bioenergetics, redox homeostasis and central carbon metabolism. Brain Res. Bull. 133, 12-30.

Baquero, M., Martín, N., 2015. Depressive symptoms in neurodegenerative diseases. World J. Clin. Cases 3, 682-693.

Canto, C., Auwerx, J., 2009. PGC-1alpha, SIRT1 and AMPK, an energy sensing network that controls energy expenditure. Curr. Opin. Lipidol. 20, 98-105.

Cao, D., Wang, M., Qiu, X., et al., 2015. Structural basis for allosteric, substrate-dependent stimulation of SIRT1 activity by resveratrol. Genes Dev. 29, 1316-1325.

Chang, J.R., Ghafouri, M., Mukerjee, R., et al., 2012. Role of p53 in neurodegenerative diseases. Neurodegener. Dis. 9, 68-80.

Chen, G.Y., Nuñez, G., 2010. Sterile inflammation: sensing and reacting to damage. Nat. Rev. Immunol. 10, 826-837.

Chen, J., Zhou, Y., Mueller-Steiner, S., et al., 2005. SIRT1 protects against microglia-dependent amyloid-beta toxicity through inhibiting NF-kappaB signaling. J. Biol. Chem. 280, 40364-40374.

Chen, X., Wales, P., Quinti, L., et al., 2015. The sirtuin-2 inhibitor AK7 is neuroprotective in models of Parkinson's disease but not amyotrophic lateral sclerosis and cerebral ischemia. PLoS One 10, e0116919.

Chen, Z., Zhai, Y., Zhang, W., et al., 2015. Single nucleotide polymorphisms of the sirtuin 1 (SIRT1) gene are associated with age-related macular degeneration in Chinese han individuals: a case-control pilot study. Medicine 94. e2238-e2238.

Cheng, C.K., Luo, J.Y., Lau, C.W., et al., 2019. Pharmacological basis and new insights of resveratrol action in the cardiovascular system. Br. J. Pharmacol..

Chopra, V., Quinti, L., Kim, J., et al., 2012. The sirtuin 2 inhibitor AK-7 is neuroprotective in Huntington's disease mouse models. Cell Rep. 2, 1492-1497.

Daly, A. k, 2010. Pharmacogenetics and human genetic polymorphisms. Biochem. J. 429, 435-449.

De Sá Coutinho, D., Pacheco, M.T., Frozza, R.L., et al., 2018. Anti-inflammatory effects of resveratrol: mechanistic insights. Int. J. Mol. Sci. 19, 1812.

Diaz-Ruiz, C., Rodriguez-Perez, A.I., Beiroa, D., et al., 2015. Reciprocal regulation between sirtuin-1 and angiotensin-II in the substantia nigra: implications for aging and neurodegeneration. Oncotarget 6, 26675-26689.

Diz, D.I., Varagic, J., Groban, L., 2008. Aging and the brain renin-angiotensin system: relevance to age-related decline in cardiac function. Future Cardiol. 4, 237-245.

Donmez, G., Outeiro, T.F., 2013. SIRT1 and SIRT2: emerging targets in neurodegeneration. EMBO Mol. Med. 5, 344-352.

Du, Y., Hu, H., Hua, C., et al., 2018. Tissue distribution, subcellular localization, and enzymatic activity analysis of human SIRT5 isoforms. Biochem. Biophys. Res. Commun. 503, 763-769.

Feigin, V.L., Nichols, E., Alam, T., et al, 2019. Global, regional, and national burden of neurological disorders, 1990-2016: a systematic analysis for the Global Burden of Disease Study 2016. Lancet Neurol. 18, 459-480.

Feng, X., Liang, N., Zhu, D., et al., 2013. Resveratrol inhibits beta-amyloid-induced neuronal apoptosis through regulation of SIRT1-ROCK1 signaling pathway. PLoS One 8 , e59888.

Ferretta, A., Gaballo, A., Tanzarella, P., et al., 2014. Effect of resveratrol on mitochondrial function: implications in parkin-associated familiar Parkinson's disease. Biochim. Biophys. Acta 1842, 902-915.

Figarska, S.M., Vonk, J.M., Boezen, H.M., 2013. SIRT1 polymorphism, long-term survival and glucose tolerance in the general population. PLoS One 8, e58636.

Finkel, T., Deng, C.X., Mostoslavsky, R., 2009. Recent progress in the biology and physiology of sirtuins. Nature 460, 587-591.

Frozza, R.L., Bernardi, A., Hoppe, J.B., et al., 2013. Lipid-core nanocapsules improve the effects of resveratrol against Abeta-induced neuroinflammation. J. Biomed. Nanotechnol. 9, 2086-2104.

Gan, L., Mucke, L., 2008. Paths of convergence: sirtuins in aging and neurodegeneration. Neuron 58, 10-14.

Gertz, M., Fischer, F., Nguyen, G.T.T., et al., 2013. Ex-527 inhibits Sirtuins by exploiting their unique NAD+-dependent deacetylation mechanism. Proc. Natl. Acad. Sci. U. S. A. 110, E2772-E2781.

Glick, D., Barth, S., Macleod, K.F., 2010. Autophagy: cellular and molecular mechanisms. J. Pathol. 221, 3-12.

Grozinger, C.M., Chao, E.D., Blackwell, H.E., et al., 2001. Identification of a class of small molecule inhibitors of the sirtuin family of NAD-dependent deacetylases by phenotypic screening. J. Biol. Chem. 276, 38837-38843. 
Guan, Q., Wang, M., Chen, H., et al., 2016. Aging-related 1-methyl-4-phenyl-1,2,3,6-tetrahydropyridine-induced neurochemial and behavioral deficits and redox dysfunction: improvement by AK-7. Exp. Gerontol. 82, 19-29.

Guo, Y.J., Dong, S.Y., Cui, X.X., et al., 2016. Resveratrol alleviates MPTP-induced motor impairments and pathological changes by autophagic degradation of alpha-synuclein via SIRT1-deacetylated LC3. Mol. Nutr. Food Res. 60, 2161-2175.

Harry, G.J., Christian, L.D.H., A, M.C., et al., 2008. Tumor necrosis factor p55 and p75 receptors are involved in chemical-induced apoptosis of dentate granule neurons. J. Neurochem. 106, 281-298.

Hay, S.I., Abajobir, A.A., Abate, K.H., et al., 2017. Global, regional, and national disability-adjusted life-years (DALYs) for 333 diseases and injuries and healthy life expectancy (HALE) for 195 countries and territories, 1990-2016: a systematic analysis for the Global Burden of Disease Study 2016. Lancet 390, 1260-1344.

Henderson, B.W., Gentry, E.G., Rush, T., et al., 2016. Rho-associated protein kinase 1 (ROCK1) is increased in Alzheimer's disease and ROCK1 depletion reduces amyloid- $\beta$ levels in brain. J. Neurochem. 138, 525-531.

Hou, X., Rooklin, D., Fang, H., et al., 2016. Resveratrol serves as a protein-substrate interaction stabilizer in human SIRT1 activation. Sci. Rep. 6. 38186-38186.

Hu, J., Jing, H., Lin, H., 2014. Sirtuin inhibitors as anticancer agents. Future Med. Chem. 6, 945-966.

Huang, W.-J., Zhang, X.I.A., Chen, W.-W., 2016. Role of oxidative stress in Alzheimer's disease. Biomed. Rep. 4, 519-522.

Jellinger, K.A., 2010. Basic mechanisms of neurodegeneration: a critical update. J. Cell Mol. Med. 14, 457-487.

Jesko, H., Wencel, P., Strosznajder, R.P., et al., 2017. Sirtuins and their roles in brain aging and neurodegenerative disorders. Neurochem. Res. 42, 876-890.

Jiang, Y., Liu, J., Chen, D., et al., 2017. Sirtuin inhibition: strategies, inhibitors, and therapeutic potential. Trends Pharmacol. Sci. 38, 459-472.

Jimenez-Sanchez, M., Licitra, F., Underwood, B.R., et al., 2017. Huntington's disease: mechanisms of pathogenesis and therapeutic strategies. Cold Spring Harbor Perspect. Med. 7. a024240.

Khan, R.S., Fonseca-Kelly, Z., Callinan, C., et al., 2012. SIRT1 activating compounds reduce oxidative stress and prevent cell death in neuronal cells. Front. Cell. Neurosci. 6, 63.

Kim, D., Nguyen, M.D., Dobbin, M.M., et al., 2007. SIRT1 deacetylase protects against neurodegeneration in models for Alzheimer's disease and amyotrophic lateral sclerosis. EMBO J. 26, 3169-3179.

Kritsilis, M., S, V.R., Koutsoudaki, P.N., et al., 2018. Ageing, cellular senescence and neurodegenerative disease. Int. J. Mol. Sci. 19

Kupis, W., Pałyga, J., Tomal, E., et al., 2016. The role of sirtuins in cellular homeostasis. J. Physiol. Biochem. 72, 371-380.

Landry, J., Sutton, A., Tafrov, S.T., et al., 2000. The silencing protein SIR2 and its homologs are NAD-dependent protein deacetylases. Proc. Natl. Acad. Sci. U. S. A. 97, 5807-5811.

Li, L., Sun, Q., Li, Y., et al., 2015. Overexpression of SIRT1 induced by resveratrol and inhibitor of miR-204 suppresses activation and proliferation of microglia. J. Mol. Neurosci. 56, 858-867.

Li, X.M., Zhou, M.T., Wang, X.M., et al., 2014. Resveratrol pretreatment attenuates the isoflurane-induced cognitive impairment through its anti-inflammation and -apoptosis actions in aged mice. J. Mol. Neurosci. 52, 286-293.

Luthi-Carter, R., Taylor, D.M., Pallos, J., et al., 2010. SIRT2 inhibition achieves neuroprotection by decreasing sterol biosynthesis. Proc. Natl. Acad. Sci. U. S. A. 107, 7927-7932.

Lv, Y., Lin, S., Peng, F., 2017. SIRT1 gene polymorphisms and risk of lung cancer. Cancer Manag. Res. 9, 381-386.

Lyman, M., Lloyd, D.G., Ji, X.M., et al., 2014. Neuroinflammation: the role and consequences. Neurosci. Res. 79, 1-12.

Mai, A., Massa, S., Lavu, S., et al., 2005. Design, synthesis, and biological evaluation of sirtinol analogues as class III histone/protein deacetylase (sirtuin) inhibitors. J. Med. Chem. 48, 7789-7795.

Mancuso, R., Del Valle, J., Modol, L., et al., 2014. Resveratrol improves motoneuron function and extends survival in SOD1(G93A) ALS mice. Neurotherapeutics 11, 419-432.

Markert, C.D., Kim, E., Gifondorwa, D.J., et al., 2010. A single-dose resveratrol treatment in a mouse model of amyotrophic lateral sclerosis. J. Med. Food 13, 1081-1085.

Michan, S., Sinclair, D., 2007. Sirtuins in mammals: insights into their biological function. Biochem. J. 404, 1-13.

Michishita, E., Park, J.Y., Burneskis, J.M., et al., 2005. Evolutionarily conserved and nonconserved cellular localizations and functions of human SIRT proteins. Mol. Biol. Cell $16,4623-4635$.

Min, S.W., Cho, S.H., Zhou, Y., et al., 2010. Acetylation of tau inhibits its degradation and contributes to tauopathy. Neuron 67, 953-966.

Min, S.W., Sohn, P.D., Cho, S.H., et al., 2013. Sirtuins in neurodegenerative diseases: an update on potential mechanisms. Front. Aging Neurosci. 5, 9.

Mogi, M., Iwanami, J., Horiuchi, M., 2012. Roles of brain angiotensin II in cognitive function and dementia. Int. J. Hypertens. 2012. 169649.

Moorthi, P., Premkumar, P., Priyanka, R., et al., 2015. Pathological changes in hippocampal neuronal circuits underlie age-associated neurodegeneration and memory loss: positive clue toward SAD. Neuroscience 301, 90-105.

Moussa, C., Hebron, M., Huang, X., et al., 2017. Resveratrol regulates neuro-inflammation and induces adaptive immunity in Alzheimer's disease. J. Neuroinflammation 14.

Mudo, G., Makela, J., Di Liberto, V., et al., 2012. Transgenic expression and activation of PGC-1alpha protect dopaminergic neurons in the MPTP mouse model of Parkinson's disease. Cell. Mol. Life Sci. 69, 1153-1165.
Nakagawa, T., Guarente, L., 2014. SnapShot: sirtuins, NAD, and aging. Cell Metabol. 20. 192-192.e1.

Nicoll, J., Holmes, C., Perry, V.H., 2010. Microglia in neurodegenerative disease. Nat. Rev. Neurol. 6, 193-201.

Niedzielska, E., Smaga, I., Gawlik, M., et al., 2016. Oxidative stress in neurodegenerative diseases. Mol. Neurobiol. 53, 4094-4125.

Nogueiras, R., Habegger, K.M., Chaudhary, N., et al., 2012. Sirtuin 1 and sirtuin 3: physiological modulators of metabolism. Physiol. Rev. 92, 1479-1514.

Outeiro, T.F., Kontopoulos, E., Altmann, S.M., et al., 2007. Sirtuin 2 inhibitors rescue alpha-synuclein-mediated toxicity in models of Parkinson's disease. Science 317, 516-519.

Ovesna, Z., Horvathova-Kozics, K., 2005. Structure-activity relationship of trans-resveratrol and its analogues. Neoplasma 52, 450-455.

Pandey, A.K., Bhattacharya, P., Shukla, S.C., et al., 2015. Resveratrol inhibits matrix metalloproteinases to attenuate neuronal damage in cerebral ischemia: a molecular docking study exploring possible neuroprotection. Neural Regen. Res. 10, 568-575.

Polito, L., Kehoe, P.G., Davin, A., et al., 2013. The SIRT2 polymorphism rs10410544 and risk of Alzheimer's disease in two Caucasian case-control cohorts. Alzheimer's Dementia 9, 392-399.

Porquet, D., Casadesus, G., Bayod, S., et al., 2013. Dietary resveratrol prevents Alzheimer's markers and increases life span in SAMP8. Age 35, 1851-1865.

Ransohoff, R.M., 2016. How neuroinflammation contributes to neurodegeneration. Science 353, 777-783.

Rizk, S.M., Shahin, N.N., Shaker, O.G., 2016. Association between SIRT1 gene polymorphisms and breast cancer in Egyptians. PLoS One 11, e0151901.

Rock, K.L., Latz, E., Ontiveros, F., et al., 2010. The sterile inflammatory response. Annu. Rev. Immunol. 28, 321-342.

Rosenberg, G.A., 2002. Matrix metalloproteinases in neuroinflammation. Glia 39, 279-291.

Scuderi, C., Stecca, C., Bronzuoli, M.R., et al., 2014. Sirtuin modulators control reactive gliosis in an in vitro model of Alzheimer's disease. Front. Pharmacol. 5, 89.

Shaw, G., 2013. Polymorphism and single nucleotide polymorphisms (SNPs). BJU Int. 112, 664-665.

Shi, C.S., Shenderov, K., Huang, N.N., et al., 2012. Activation of autophagy by inflammatory signals limits IL-1beta production by targeting ubiquitinated inflammasomes for destruction. Nat. Immunol. 13, 255-263.

Shih, R.-H., Wang, C.-Y., Yang, C.-M., 2015. NF-kappaB signaling pathways in neurological inflammation: a mini review. Front. Mol. Neurosci. 8, 77.

Shimoyama, Y., Mitsuda, Y., Tsuruta, Y., et al., 2012. SIRTUIN 1 gene polymorphisms are associated with cholesterol metabolism and coronary artery calcification in Japanese hemodialysis patients. J. Ren. Nutr. 22, 114-119.

Shoba, B., Lwin, Z.M., Ling, L.S., et al., 2009. Function of sirtuins in biological tissues. Anat. Rec. 292, 536-543.

Silva, D.F., Esteves, A.R., Oliveira, C.R., et al., 2016. Mitochondrial metabolism powe SIRT2-dependent deficient traffic causing alzheimer's-disease related pathology. Mol. Neurobiol. 54, 4021-4040.

Skovronsky, D.M., Lee, V.M.Y., Trojanowski, J.Q., 2006. Neurodegenerative diseases: new concepts of pathogenesis and their therapeutic implications. Annu. Rev. Pathol. 1, 151-170.

Smith, M.R., Syed, A., Lukacsovich, T., et al., 2014. A potent and selective Sirtuin 1 in hibitor alleviates pathology in multiple animal and cell models of Huntington's disease. Hum. Mol. Genet. 23, 2995-3007.

Song, L., Chen, L., Zhang, X., et al., 2014. Resveratrol ameliorates motor neuron degen eration and improves survival in SOD1(G93A) mouse model of amyotrophic latera sclerosis. BioMed Res. Int. 2014. 483501.

Spires-Jones, T.L., Attems, J., Thal, D.R., 2017. Interactions of pathological proteins in neurodegenerative diseases. Acta Neuropathol. 134, 187-205.

Sussmuth, S.D., Haider, S., Landwehrmeyer, G.B., et al., 2015. An exploratory double-blind, randomized clinical trial with selisistat, a SirT1 inhibitor, in patients with Huntington's disease. Br. J. Clin. Pharmacol. 79, 465-476.

Sweeney, G., Song, J., 2016. The association between PGC-1 $\alpha$ and Alzheimer's disease. Anat. Cell Biol. 49, 1-6.

Tang, B.L., 2017. Sirtuins as modifiers of Parkinson's disease pathology. J. Neurosci. Res. 95, 930-942.

Valle, C., Salvatori, I., Gerbino, V., et al., 2014. Tissue-specific deregulation of selected HDACs characterizes ALS progression in mouse models: pharmacological characterization of SIRT1 and SIRT2 pathways. Cell Death Dis. 5, e1296.

Wang, B., Zhang, Y., Cao, W., et al., 2016. SIRT2 plays significant roles in lipopolysaccharides-induced neuroinflammation and brain injury in mice. Neurochem. Res. 41, 2490-2500.

Wang, J., Zhang, Y., Tang, L., et al., 2011. Protective effects of resveratrol through the up-regulation of SIRT1 expression in the mutant hSOD1-G93A-bearing motor neuron-like cell culture model of amyotrophic lateral sclerosis. Neurosci. Lett. 503, 250-255.

Wang, X., Guan, Q., Wang, M., et al., 2015. Aging-related rotenone-induced neurochemical and behavioral deficits: role of SIRT2 and redox imbalance, and neuroprotection by AK-7. Drug Des. Dev. Ther. 9, 2553-2563.

Wong, D., Dorovini-Zis, K., Vincent, S.R., 2004. Cytokines, nitric oxide, and cGMP modulate the permeability of an in vitro model of the human blood-brain barrier. Exp. Neurol. 190, 446-455.

Wright, J.W., Harding, J.W., 2004. The brain angiotensin system and extracellular matrix molecules in neural plasticity, learning, and memory. Prog. Neurobiol. 72, 263-293.

Xia, M., Yu, J.-T., Miao, D., et al., 2014. SIRT2 polymorphism rs10410544 is associated with Alzheimer's disease in a Han Chinese population. J. Neurol. Sci. 336, 48-51.

Ye, J., Liu, Z., Wei, J., et al., 2013. Protective effect of SIRT1 on toxicity of microglial-derived factors induced by LPS to PC12 cells via the p53-caspase-3-dependent apoptotic pathway. Neurosci. Lett. 553, 72-77. 
Yuan, F., Xu, Z.M., Lu, L.Y., et al., 2016. SIRT2 inhibition exacerbates neuroinflammation and blood-brain barrier disruption in experimental traumatic brain injury by enhancing NF-кB p65 acetylation and activation. J. Neurochem. 136, 581-593. 